\title{
Comparison of in vitro maturation and in vitro fertilization for polycystic ovary syndrome patients: a systematic review and meta-analysis
}

\author{
Yalan Xut ${ }^{1,2,3,4,5}$, Jie Qiao ${ }^{1,2,3,4,5}$ ^ \\ ${ }^{1}$ Centre for Reproductive Medicine, Department of Obstetrics and Gynecology, Peking University Third Hospital, Beijing, China; ${ }^{2}$ National \\ Clinical Research Centre for Obstetrics and Gynecology, Beijing, China; ${ }^{3}$ Key Laboratory of Assisted Reproduction (Peking University), Ministry \\ of Education, Beijing, China; ${ }^{4}$ Beijing Key Laboratory of Reproductive Endocrinology and Assisted Reproductive Technology, Beijing, China; \\ ${ }^{5}$ Research Units of Comprehensive Diagnosis and Treatment of Oocyte Maturation Arrest, Chinese Academy of Medical Sciences, Beijing, China \\ Contributions: (I) Conception and design: Both authors; (II) Administrative support: Both authors; (III) Provision of study materials or patients: \\ Both authors; (IV) Collection and assembly of data: Both authors; (V) Data analysis and interpretation: Both authors; (VI) Manuscript writing: Both \\ authors; (VII) Final approval of manuscript: Both authors. \\ Correspondence to: Jie Qiao. Centre for Reproductive Medicine, Department of Obstetrics and Gynecology, Peking University Third Hospital, No. 49 \\ North Huayuan Road, Haidian District, Beijing 100191, China. Email: jie.qiao@263.net.
}

Background: There are two common treatments for polycystic ovary syndrome (PCOS): in vitro fertilization (IVF) and in vitro maturation (IVM). Our study aimed to assess the clinical effects and safety of IVM versus IVF for PCOS.

Methods: We searched randomized controlled trials and retrospective cohort studies comparing IVM versus IVF for PCOS. Data were extracted from eligible studies. We sought to evaluate fertilization rate, clinical pregnancy rate, live birth rate, and miscarriage. Results were expressed as risk ratio (RR) with $95 \%$ confidence intervals (CIs).

Results: Eight studies with a total of 1,579 patients were included in the present study. According to the heterogeneity analysis, there were no differences between the IVM group and IVF group in terms of fertilization rate, clinical pregnancy rate and miscarriage. Additionally, the IVF group had a higher live birth rate than the IVM group (overall $\mathrm{P}=0.0007$ ). Sensitivity analysis and funnel plot showed that our study was robust and based on the funnel plot this article had low publication bias.

Discussion: The findings of the present study indicated that IVM had similar clinical effects compared with IVF in patients with PCOS. However, IVM might be a suitable option for PCOS in terms of cost and successful pregnancy rate.

Keywords: In vitro maturation (IVM); in vitro fertilization (IVF); polycystic ovary syndrome (PCOS); metaanalysis

Submitted May 15, 2021. Accepted for publication Jul 02, 2021.

doi: 10.21037/atm-21-3037

View this article at: https://dx.doi.org/10.21037/atm-21-3037

\section{Introduction}

In vitro fertilization (IVF) is used for the treatment of infertility. During IVF, eggs that have developed to near ovulation are removed from the ovary and placed in a culture medium to fertilize them $(1,2)$. Fertilized eggs that have grown to a certain degree are placed into the uterus. However, ovarian hyperstimulation syndrome (OHSS) may occur, especially in patients with polycystic ovary syndrome

\footnotetext{
^ ORCID: 0000-0002-4131-2890.
} 
(PCOS) (3). A potentially helpful alternative for women with PCOS is to remove mature oocytes from small antral follicles early, without hormone stimulation, and then perform in vitro maturation (IVM) of oocytes (4). IVM in patients with PCOS can eliminate the risk of OHSS and the cost, but when compared with IVF, the success rate of IVM is lower (5).

PCOS is the most common endocrine abnormality in women of childbearing age, affecting $6.6-8 \%$ of women in this age group. It is the leading cause of anovulatory infertility, and is characterized by chronic anovulation, hyperandrogenemia, and polycystic ovarian disease $(6,7)$. PCOS is a complex heterogeneous disorder of uncertain etiology and the diagnosis of PCOS is based on the onset of puberty, abnormal menstruation and ovulation, hirsutism, increased serum luteinizing hormone (LH) and LH/ follicle-stimulating hormone (FSH) ratio, combined with high androgen level, polycystic ovary signs in ultrasound examination, and exclusion of other similar diseases (7). Women with PCOS have a significantly increased risk of OHSS. In assisted conception, OHSS is an iatrogenic complication of ovulation induction and ovarian stimulation cycles (8). In severe cases, it may be life threatening (9). It is characterized by enlarged ovarian cysts and rapid transfer of fluid from the vascular cavity to the third space (10).

IVM was first used in 1991 for successful pregnancies and the delivery of live babies. Since then, several variants of IVM have been successfully used as alternatives to IVF in different patient groups, with varying success rates, but they have not been widely accepted $(11,12)$. IVM is an optionassisted reproductive technology. In IVM, small follicles with a diameter of $2-10 \mathrm{~mm}$ are removed without prior administration of human chorionic gonadotropin and then mature in vitro (13). Compared with IVF, this method has several advantages, including no (or minimal) controlled ovarian stimulation, improved convenience, and lower monitoring burden (14). IVM is a successful and widely used assisted reproductive technology in advanced livestock breeding. IVM includes the extraction of immature oocytes in the foaming stage and in vitro culturing of entire cumulus-oocyte complexes until metaphase II, then routine IVF or intracytoplasmic injection (15).

In the present study, meta-analysis was used to combine the results of different research, and several evaluation outcomes were compared with intervention methods to provide a choice for PCOS patients. In this study, we compared two treatment for PCOS in several aspects from the start fertilization to the end live birth, and included several recent published articles to further analyze the effects of IVM.

We present the following article in accordance with the PRISMA reporting checklist (available at https://dx.doi. org/10.21037/atm-21-3037).

\section{Methods}

\section{Literature search}

From January 2000 to March 2021, we carried out a systematic search on databases, including PubMed, Embase, Cochrane Library, and China National Knowledge Infrastructure, using the following keywords: in vitro maturation or IVM, in vitro fertilization or IVF, and polycystic ovary syndrome or PCOS. The search term was combined using the Boolean operator "and". The language of publication did not restrict the literature search. We manually searched the reference lists of the retrieved articles to find any relevant research that the search strategy might have missed.

\section{Inclusion and exclusion criteria}

We included terms that met the following inclusion criteria: (I) PCOS patients; (II) the experimental group was treated with IVM and the control group was treated with IVF; (III) indicators to evaluate the effectiveness and safety of the IVM group and the IVF group; and (IV) full-text articles.

We excluded research for the following reasons: (I) the research did not meet the inclusion criteria; (II) unreported or unavailable results of interest; and (III) reviews, abstracts, and repeated publications.

\section{Literature screening and data extraction}

After screening the titles and abstracts of possible eligible studies, two reviewers independently read the full text and extracted relevant data, including the first author's name, study design, sample size, patients' age, and sex, year of onset, study duration, and the main result. The methodological quality of the included studies was assessed using the Cochrane risk-of-bias assessment tool.

\section{Statistical methods}

Meta-analysis was performed using Review Manager 5.4 (Cochrane). $\mathrm{P}<0.05$ or $\mathrm{I}^{2}>50 \%$ indicated that there was a 


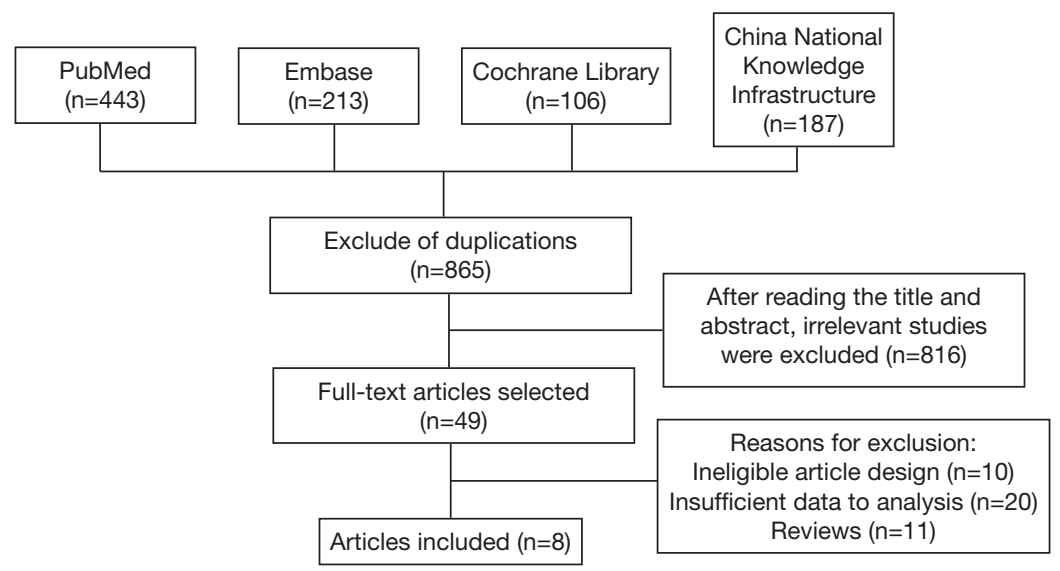

Figure 1 Flowchart of inclusion.

certain degree of heterogeneity between the studies, and the random-effects model was used for analysis. $\mathrm{P} \geq 0.05$ and $\mathrm{I}^{2} \leq 50 \%$ indicated that there was no heterogeneity between the lessons or the heterogeneity was small, and the fixedeffects model for analysis. Risk ratio (RR) was used to analyze binary variables, mean difference (MD) and $95 \%$ confidence interval (CI) were used to analyze continuous variables, and U-test was used to test hypotheses. Sensitivity analysis was performed by excluding individual studies.

\section{Results}

\section{Literature search results}

We identified 865 studies following our electronic search. Following abstract reading and analysis, 816 articles were excluded because they did not meet the study's inclusion criteria. Of these, 49 articles were further excluded because of different study designs or insufficient data available. Finally, eight papers met the selection criteria and were included in the meta-analysis (16-23). The search process and full inclusion/exclusion criteria are shown in Figure 1.

\section{Characteristics of the selected literature}

The characteristics of the eight studies included in our meta-analysis are shown in Table 1 . The published years were from 2011 to 2020 . The studies included 1,579 patients (753 patients receiving IVM and 826 patients receiving IVF). The primary outcomes were fertilization rate, clinical pregnancy rate, live birth, and miscarriage.

\section{Methodological quality of the selected studies}

According to the Cochrane risk-of-bias assessment tool, we assessed the methodological quality of the included studies for risk of bias. We found high selection bias and other biases in three different studies (Figure 2). A summary of the risk-of-bias assessment of the eight included studies is shown in Figure 3.

\section{Meta-analysis results}

\section{Heterogeneity analysis of fertilization rate between IVM and IVF}

To analyze the difference in fertilization rate between the IVM and the IVF groups, we performed a meta-analysis to calculate the RR using the fixed-effect model based on heterogeneity analysis. The overall RR was 0.93 with $95 \%$ CI: $0.86-1.00$. The $\mathrm{P}$ value of overall effect was 0.07 and $\mathrm{I}^{2}=48 \%$, which demonstrated that there was no significant difference in fertilization rate between the IVM and IVF groups (Figure 4).

\section{Heterogeneity analysis of clinical pregnancy rate between IVM and IVF}

Similarly, a meta-analysis for the difference in clinical pregnancy rate between IVM and IVF groups was conducted. The result showed that there was no significant difference in the clinical pregnancy rate among the two groups (RR $=0.90,95 \%$ CI: $0.79-1.01, P=0.08$, fixed-effects model), while clinical pregnancy rate among the included studies showed low homogeneous $\left(\mathrm{P}=0.19, \mathrm{I}^{2}=31 \%\right)$ (Figure 5). 
Table 1 Characteristics of studies included in the meta-analysis

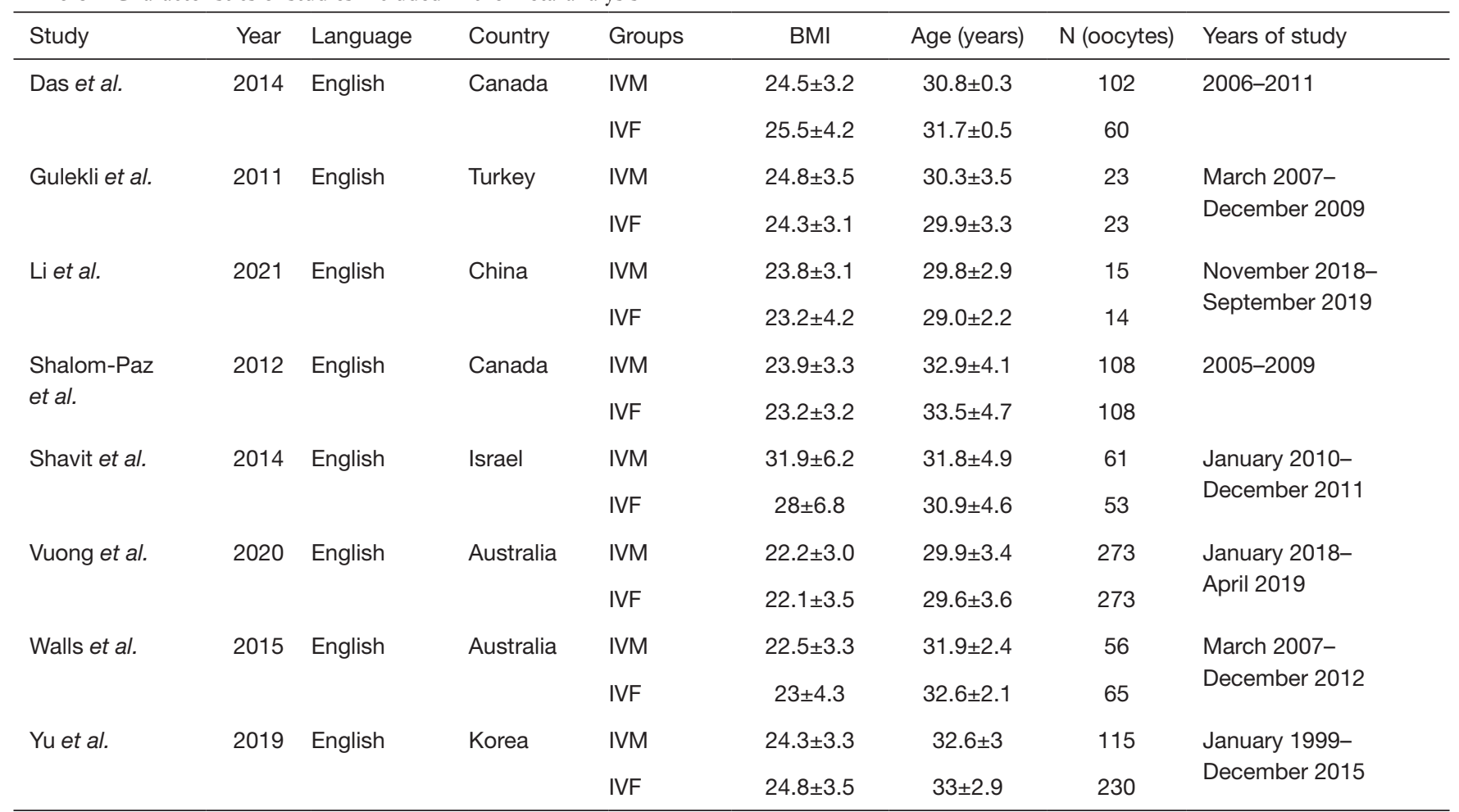

BMI, body mass index; IVF, in vitro fertilization; IVM, in vitro maturation.

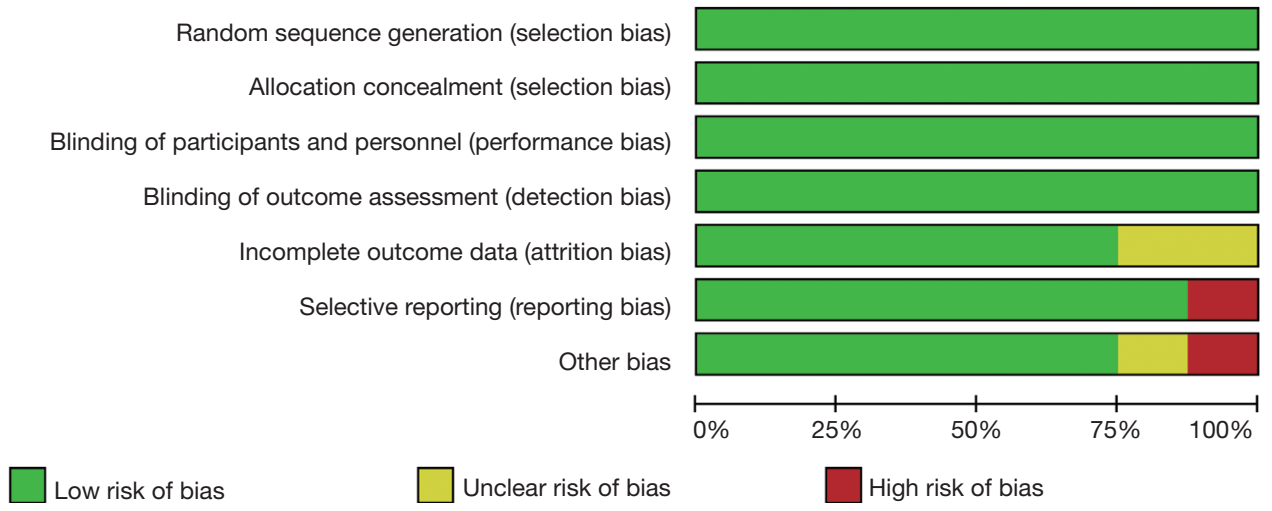

Figure 2 Assessment of the quality of the included studies: low risk of bias (green), unclear risk of bias (yellow), and high risk of bias (red).

\section{Heterogeneity analysis of live birth rate between IVM and IVF}

For live birth rate, seven studies with 1,234 patients were selected. Meta-analysis showed that, compared with the IVM group, the IVF group had a higher live birth rate (MD $=0.82$, 95\% CI: $0.70-0.94, \mathrm{P}=0.007$, fixed-effects model), with significant heterogeneity $\left(\mathrm{I}^{2}=26 \%\right.$ ) (Figure 6).

\section{Heterogeneity analysis of miscarriage between IVM and IVF}

A fixed-effects model was used to evaluate the heterogeneity of miscarriage, as insignificant heterogeneity was found among the included studies $\left(\mathrm{P}=0.40, \mathrm{I}^{2}=0 \%\right)$. The results showed that there was no difference between the IVM and IVF groups in the evaluation of miscarriage $(M D=1.27$ 


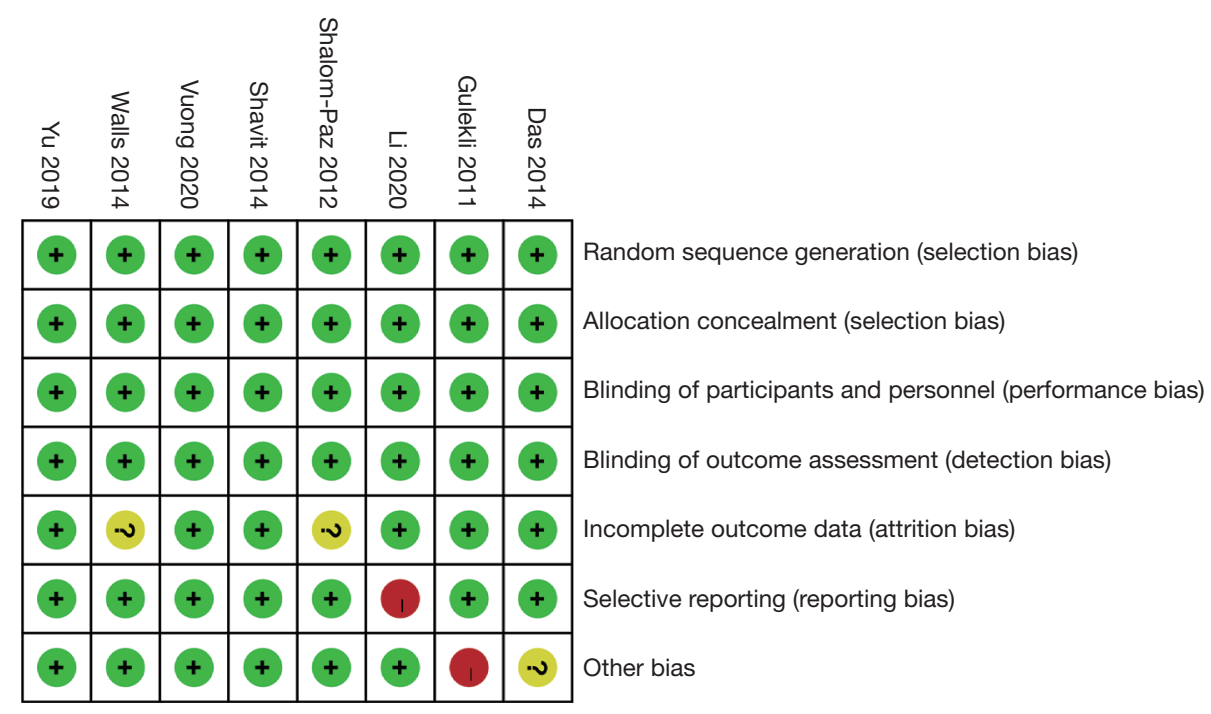

Figure 3 Quality assessment of included studies.

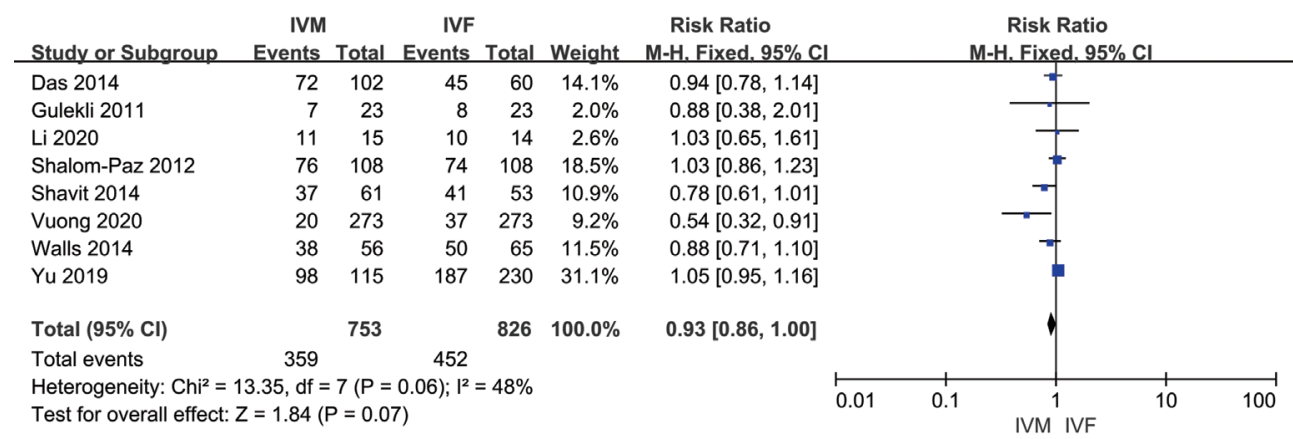

Figure 4 Forest plots of fertilization rate between IVM and IVF. IVM, in vitro maturation; IVF, in vitro fertilization; CI, confidence interval.

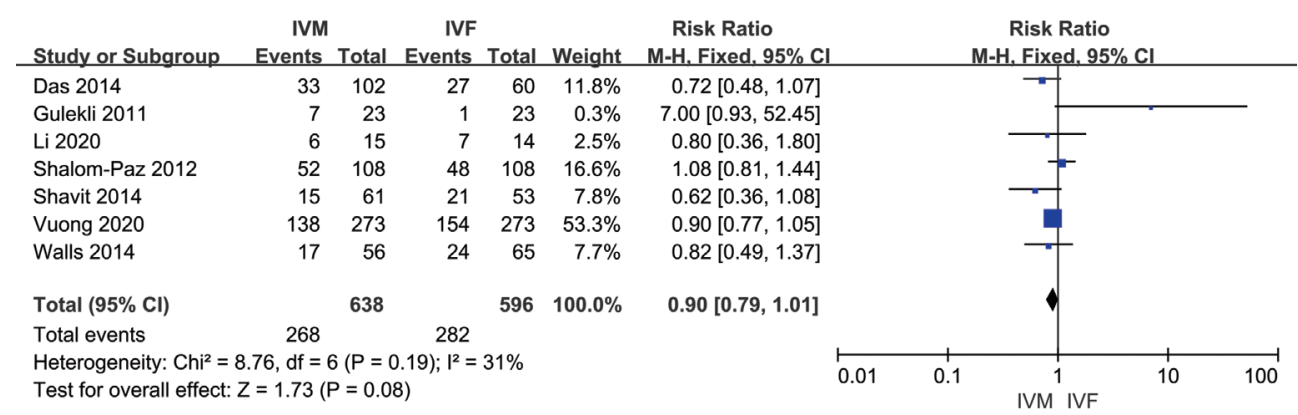

Figure 5 Forest plots of clinical pregnancy rate between IVM and IVF. IVM, in vitro maturation; IVF, in vitro fertilization; CI, confidence interval. 


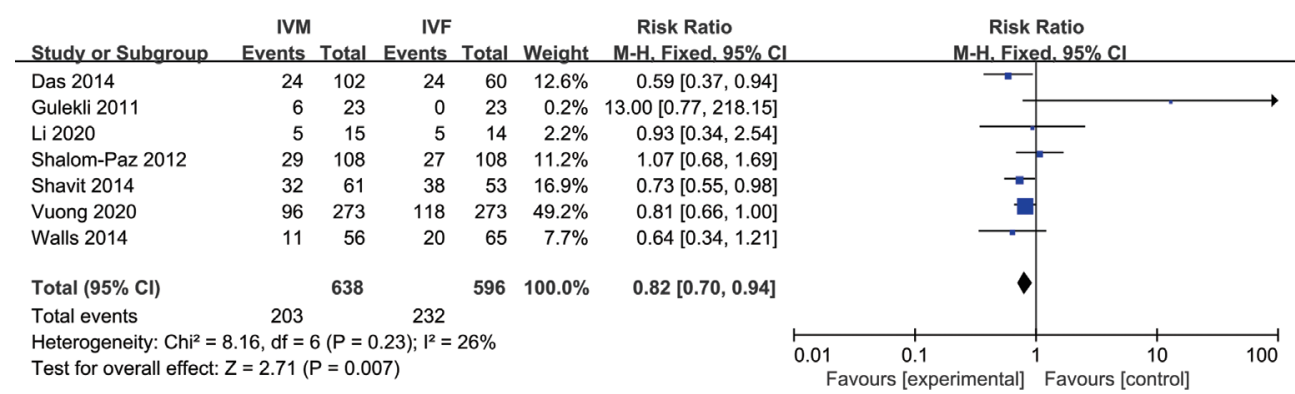

Figure 6 Forest plots of live birth rate between IVM and IVF. IVM, in vitro maturation; IVF, in vitro fertilization; CI, confidence interval.

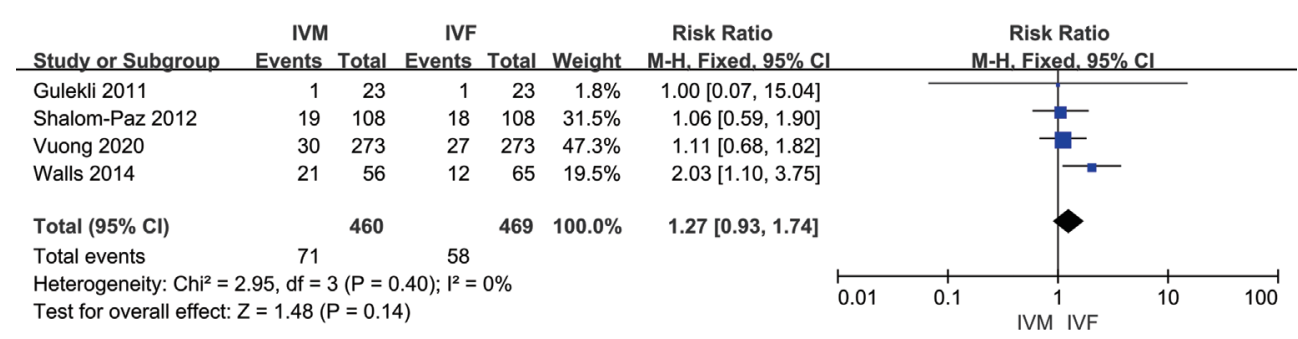

Figure 7 Forest plots of miscarriage between IVM and IVF. IVM, in vitro maturation; IVF, in vitro fertilization; CI, confidence interval.

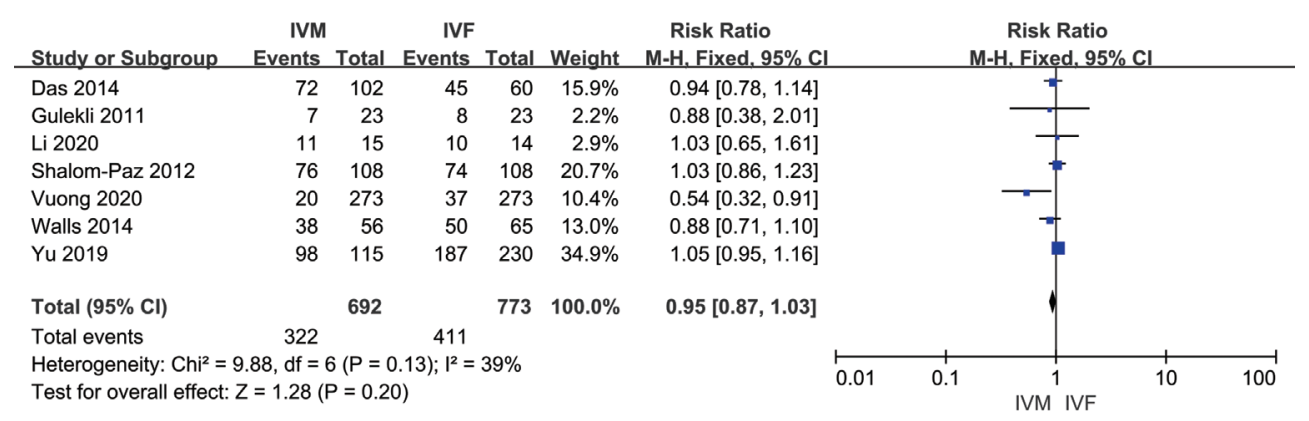

Figure 8 Sensitivity analysis forest plots of fertilization rate between IVM and IVF. IVM, in vitro maturation; IVF, in vitro fertilization; CI, confidence interval.

with 95\% CI: 0.93-1.74, $\mathrm{P}=0.14$ ) (Figure 7).

\section{Results of sensitivity analysis and publication bias}

A total of eight studies reported fertilization rate. The forest plot showed no significant difference between the IVM and IVF groups ( $R R=0.93$ with $95 \%$ CI: $0.86-1.00$ ), while the $\mathrm{P}$ value of overall effect was 0.07 and $\mathrm{I}^{2}=48 \%$ (Figure 4). We performed a sensitivity analysis by removing Shavit et al.'s 2014 study, and $\mathrm{I}^{2}$ changed from $48 \%$ to $39 \%$, which indicated that the results of included articles were robust (Figure 8).

We performed a funnel plot to evaluate the publication bias for recovery rate qualitatively (Figure 9). The Figure 9 showed that the shape was symmetric. The $\mathrm{P}$ value of the Egger test was 0.519 , which indicated that no significant publication bias existed in this meta-analysis.

\section{Discussion}

\section{Difference between IVM and IVF among PCOS patients}

In the present study, eight studies met the inclusion criteria to evaluate the difference between IVM and IVF. A meta-analysis of these studies showed that there were differences in the live birth rate, and the IVM group 


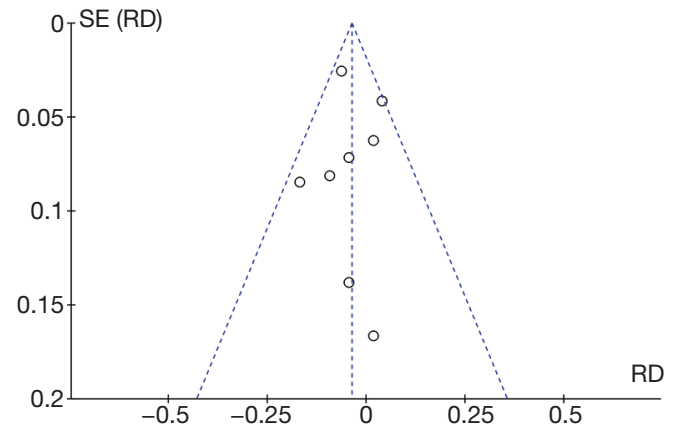

Figure 9 Funnel plot: Each circle represents one included article. If the figure symmetry is good, the publication bias is small.

had a lower live birth rate than the IVF group. When comparing the fertilization rate, clinical pregnancy rate, and miscarriage, there was no difference between the IVM and IVF groups. These results were consistent with Alm's research that the maturity obtained using IVM stimulation program was lower than the standard IVF cycle during ovariectomy. The cytoplasmic immaturation might cause the live birth rate to be significantly lower than that of the standard IVF group (24).

\section{IVM is a suitable method for treating PCOS compared with IVF}

When gonadotropin stimulation was ineffective for women undergoing IVF, this was a challenge for reproductive doctors. Several studies have explored dropouts, including poor prognosis and economic or physiological burden $(25,26)$. A new study found that emotional distress and poor prognosis were the main reasons for IVF withdrawal. There are currently different methods to help patients undergoing IVF, including changing the ovarian stimulation program, natural cycle IVF, or oocyte donation (27). IVM of immature oocytes extracted from unstimulated ovaries is a relatively new assisted reproductive technology that has shown promising results in women with polycystic ovaries $(28,29)$. Although the classic indication for IVM is PCOS, it is also suitable for other symptoms, including inadequate response, preservation of fertility in cancer patients, regular responders with a history of poor oocyte/embryo quality, and oocyte donation. The cryopreservation of immature oocytes is the most important method for fertilization $(30,31)$.

In terms of IVM safety, some researchers believe that, compared with mature oocytes in vivo, oocytes matured in vitro have the characteristics of multiple cells and molecular defects, which lead to lower development potential of IVM oocytes $(32,33)$. Compared with conventional IVF, IVM involves several additional steps, including isolation, processing, and oocyte culture (34). Compared with mature oocytes in vivo, these steps might exert environmental pressure on oocytes. The spindle change is also related to prolonged maturation time in vitro, making the cells susceptible to aneuploidy or maturation arrest (35). Finally, it was reported that IVM could cause permanent changes in the expression of imprinted genes. DNA methylation played a vital role in regulating embryo growth and establishing genomic imprinting. Incomplete methylation in maternal during in vitro culture might lead to severe consequences, such as Beckwith-Wiedemann syndrome, Angelman syndrome, and Silver-Russell syndrome (36). Some previous studies have shown that epigenetic abnormalities are more likely to occur following IVM (37).

It was also reported that, compared with the standard IVF group, the IVM group had a lower maturity rate and a lower average fertilization rate; however, the difference in the average number of oocytes collected or matured per patient was not statistically significant (38). The main goal of the IVM cycle is to enable oocytes to complete meiosis during the mature culture process, and obtained the nucleus and cytoplasm. After that, it can achieve fertilization, embryonic development, and ultimately healthy live birth (39).

\section{Limitations}

The present study has some limitations. First, the efficacy indicators were not comprehensive, but results pertaining to length of hospitalization, complications, mortality, safety indicators, and other indicators were lacking. Second, different studies had different follow-up times, affecting the final results.

\section{Conclusions}

IVM is essential for treating PCOS, but there is no significant difference in clinical efficacy compared with IVF. Due to the limitations in the number and quality of the research, our finding needs to be confirmed by a largesample, multicenter, follow-up controlled trial.

\section{Acknowledgments}

Funding: This study was supported financially by National Natural Science Foundation of China (No. 81730038) and 
CAMS Innovation Fund for Medical Sciences (No. 2019I2M-5-001).

\section{Footnote}

Reporting Checklist: The authors have completed the PRISMA reporting checklist. Available at https://dx.doi. org/10.21037/atm-21-3037

Conflicts of Interest: Both authors have completed the ICMJE uniform disclosure form (available at https://dx.doi. org/10.21037/atm-21-3037). The authors have no conflicts of interest to declare.

Ethical Statement: The authors are accountable for all aspects of the work in ensuring that questions related to the accuracy or integrity of any part of the work are appropriately investigated and resolved.

Open Access Statement: This is an Open Access article distributed in accordance with the Creative Commons Attribution-NonCommercial-NoDerivs 4.0 International License (CC BY-NC-ND 4.0), which permits the noncommercial replication and distribution of the article with the strict proviso that no changes or edits are made and the original work is properly cited (including links to both the formal publication through the relevant DOI and the license). See: https://creativecommons.org/licenses/by-nc-nd/4.0/.

\section{References}

1. Liu X, Wang J, Fu X, et al. Thin endometrium is associated with the risk of hypertensive disorders of pregnancy in fresh IVF/ICSI embryo transfer cycles: a retrospective cohort study of 9,266 singleton births. Reprod Biol Endocrinol 2021;19:55.

2. Metzemaekers J, Lust E, Rhemrev J, et al. Prognosis in fertilisation rate and outcome in IVF cycles in patients with and without endometriosis: a population-based comparative cohort study with controls. Facts Views Vis Obgyn 2021;13:27-34.

3. Ho CW, Chen HH, Hsieh MC, et al. Hashimoto's thyroiditis might increase polycystic ovary syndrome and associated comorbidities risks in Asia. Ann Transl Med 2020;8:684.

4. Hoffert KA, Anderson GB, Wildt DE, et al. Transition from maternal to embryonic control of development in IVM/IVF domestic cat embryos. Mol Reprod Dev
1997;48:208-15.

5. Lizzi KM, Qualls WA, Brown SC, et al. Expanding Integrated Vector Management to promote healthy environments. Trends Parasitol 2014;30:394-400.

6. Hong KH, Forman EJ, Lee H, et al. Optimizing the temperature for embryo culture in IVF: a randomized controlled trial (RCT) comparing standard culture temperature of $37 \mathrm{C}$ to the reduced more physiologic temperature of 36C. Fertil Steril 2012;98:S167.

7. Yang Z, Liu J, Collins GS, et al. Selection of single blastocysts for fresh transfer via standard morphology assessment alone and with array CGH for good prognosis IVF patients: results from a randomized pilot study. Mol Cytogenet 2012;5:24.

8. Albuz FK, Sasseville M, Lane M, et al. Simulated physiological oocyte maturation (SPOM): a novel in vitro maturation system that substantially improves embryo yield and pregnancy outcomes. Hum Reprod 2010;25:2999-3011.

9. Shalom-Paz E, Almog B, Shehata F, et al. Fertility preservation for breast-cancer patients using IVM followed by oocyte or embryo vitrification. Reprod Biomed Online 2010;21:566-71.

10. Vanderzwalmen P, Ectors F, Grobet L, et al. Aseptic vitrification of blastocysts from infertile patients, egg donors and after IVM. Reprod Biomed Online 2009;19:700-7.

11. Fadini R, Dal Canto MB, Mignini Renzini M, et al. Effect of different gonadotrophin priming on IVM of oocytes from women with normal ovaries: a prospective randomized study. Reprod Biomed Online 2009;19:343-51.

12. Broer SL, Mol BW, Hendriks D, et al. The role of antimullerian hormone in prediction of outcome after IVF: comparison with the antral follicle count. Fertil Steril 2009;91:705-14.

13. Beier JC, Keating J, Githure JI, et al. Integrated vector management for malaria control. Malar J 2008; 7 Suppl 1:S4.

14. Gnoth C, Schuring AN, Friol K, et al. Relevance of anti-Mullerian hormone measurement in a routine IVF program. Hum Reprod 2008;23:1359-65.

15. Lonergan P, Fair T, Corcoran D, et al. Effect of culture environment on gene expression and developmental characteristics in IVF-derived embryos. Theriogenology 2006;65:137-52.

16. Li X, Mu Y, Elshewy N, et al. Comparison of IVF and IVM outcomes in the same patient treated with a modified IVM protocol along with an oocytes-maturing system containing melatonin: a pilot study. Life Sci 
2021;264:118706.

17. Vuong LN, Ho VNA, Ho TM, et al. In-vitro maturation of oocytes versus conventional IVF in women with infertility and a high antral follicle count: a randomized non-inferiority controlled trial. Hum Reprod 2020;35:2537-47.

18. Yu EJ, Yoon TK, Lee WS, et al. Obstetrical, neonatal, and long-term outcomes of children conceived from in vitro matured oocytes. Fertil Steril 2019;112:691-9.

19. Walls ML, Hunter T, Ryan JP, et al. In vitro maturation as an alternative to standard in vitro fertilization for patients diagnosed with polycystic ovaries: a comparative analysis of fresh, frozen and cumulative cycle outcomes. Hum Reprod 2015;30:88-96.

20. Shavit T, Ellenbogen A, Michaeli M, et al. In-vitro maturation of oocytes vs in-vitro fertilization with a gonadotropin-releasing hormone antagonist for women with polycystic ovarian syndrome: can superiority be defined? Eur J Obstet Gynecol Reprod Biol 2014;179:46-50.

21. Das $M$, Son WY, Buckett $W$, et al. In-vitro maturation versus IVF with GnRH antagonist for women with polycystic ovary syndrome: treatment outcome and rates of ovarian hyperstimulation syndrome. Reprod Biomed Online 2014;29:545-51.

22. Shalom-Paz E, Holzer H, Son W, et al. PCOS patients can benefit from in vitro maturation (IVM) of oocytes. Eur J Obstet Gynecol Reprod Biol 2012;165:53-6.

23. Gulekli B, Kovali M, Aydiner F, et al. IVM is an alternative for patients with PCO after failed conventional IVF attempt. J Assist Reprod Genet 2011;28:495-9.

24. Alm H, Torner H, Löhrke B, et al. Bovine blastocyst development rate in vitro is influenced by selection of oocytes by brillant cresyl blue staining before IVM as indicator for glucose-6-phosphate dehydrogenase activity. Theriogenology 2005;63:2194-205.

25. Pandian Z, Templeton A, Serour G, et al. Number of embryos for transfer after IVF and ICSI: a Cochrane review. Hum Reprod 2005;20:2681-7.

26. $\mathrm{Li} \mathrm{T}, \mathrm{Vu} \mathrm{TH}, \mathrm{Ulaner} \mathrm{GA}$, et al. IVF results in de novo DNA methylation and histone methylation at an Igf2-H19 imprinting epigenetic switch. Mol Hum Reprod 2005;11:631-40.

27. Elizur SE, Aslan D, Shulman A, et al. Modified natural cycle using GnRH antagonist can be an optional treatment in poor responders undergoing IVF. J Assist Reprod Genet $2005 ; 22: 75-9$.
28. Hong JY, Yong HY, Lee BC, et al. Effects of amino acids on maturation, fertilization and embryo development of pig follicular oocytes in two IVM media. Theriogenology 2004;62:1473-82.

29. Campbell R, Taylor W. Interactive video monitoring (IVM) process. U.S. Patent Application 10/804,543. 2004-9-23.

30. Brison DR, Houghton FD, Falconer D, et al. Identification of viable embryos in IVF by non-invasive measurement of amino acid turnover. Hum Reprod 2004;19:2319-24.

31. Gerris J, De Sutter P, De Neubourg D, et al. A reallife prospective health economic study of elective single embryo transfer versus two-embryo transfer in first IVF/ ICSI cycles. Hum Reprod 2004;19:917-23.

32. Bungum $M$, Humaidan $P$, Spano $M$, et al. The predictive value of sperm chromatin structure assay (SCSA) parameters for the outcome of intrauterine insemination, IVF and ICSI. Hum Reprod 2004;19:1401-8.

33. Collins J. An international survey of the health economics of IVF and ICSI. Hum Reprod Update 2002;8:265-77.

34. Bartoov B, Berkovitz A, Eltes F, et al. Real-time fine morphology of motile human sperm cells is associated with IVF-ICSI outcome. J Androl 2002;23:1-8.

35. Bonduelle M, Liebaers I, Deketelaere V, et al. Neonatal data on a cohort of 2889 infants born after ICSI (19911999) and of 2995 infants born after IVF (1983-1999). Hum Reprod 2002;17:671-94.

36. Gutiérrez-Adán A, Granados J, Pintado B, et al. Influence of glucose on the sex ratio of bovine IVM/IVF embryos cultured in vitro. Reprod Fertil Dev 2001;13:361-5.

37. Martikainen H, Tiitinen A, Tomás C, et al. One versus two embryo transfer after IVF and ICSI: a randomized study. Hum Reprod. 2001;16:1900-3.

38. Chen Q, Kirsch GE, Zhang D, et al. Genetic basis and molecular mechanism for idiopathic ventricular fibrillation. Nature 1998;392:293-6.

39. Ericson A, Källén B. Congenital malformations in infants born after IVF: a population-based study. Hum Reprod 2001;16:504-9.

(English Language Editor: R. Scott)

Cite this article as: $\mathrm{Xu} \mathrm{Y,} \mathrm{Qiao} \mathrm{J.} \mathrm{Comparison} \mathrm{of} \mathrm{in} \mathrm{vitro}$ maturation and in vitro fertilization for polycystic ovary syndrome patients: a systematic review and meta-analysis. Ann Transl Med 2021;9(15):1235. doi: 10.21037/atm-21-3037 